\title{
INFLUÊNCIA DA FONTE DE LUZ, ESPESSURA E TRANSLUCIDEZ DA CERÂMICA NA RESISTÊNCIA DE UNIÃO E IRRADIÂNCIA
}

\section{Camila Imperador Rodrigues Alves *, Lincoln Pires Silva Borges, Lourenço Correr Sobrinho, Ana Rosa Costa.}

\section{Resumo}

Este estudo avaliou o efeito da emissão de um ou múltiplos comprimentos de onda (Radii-cal e Bluephase G2) através da cerâmica IPS e.max Press em diferentes espessuras $(0,5,1,5$ e 2,0 mm) e translucidez (HT - alta translucidez, MT - média translucidez e LT - baixa translucidez) na passagem de luz (Irradiância - $\mathrm{mW} / \mathrm{cm} 2$ ) e na resistência da união ao microcisalhamento $(\mathrm{RU} \mu \mathrm{C})$ do cimento resinoso fotoativado Variolink Esthetic LC. Noventa discos de cerâmica (12 $\mathrm{mm}$ diâmetro) foram separados em 18 grupos $(n=5)$. Um lado do disco recebeu acabamento, polimento e glaze. A irradiância $\left(\mathrm{mW} / \mathrm{cm}^{2}\right)$ foi avaliada com potenciômetro Ophir 10 $0^{\mathrm{a}}-\mathrm{V} 2-\mathrm{SH}$ imediatamente após a interposição cerâmica. A $\mathrm{RU} \mu \mathrm{C}$ foi realizada à velocidade de $1,0 \mathrm{~mm} /$ minuto até ocorrer falha. Os dados foram submetidos à Análise de Variância 3 fatores e ao teste de Tukey $(\alpha=0,05)$. Os valores de $R U \mu C(M P a)$ dos grupos $R D(37,1 \pm 6,4)$ apresentaram valores estatisticamente menores que os grupos $B L(45,3 \pm 5,8)(p=0,000)$. A espessura de $0,5 \mathrm{~mm}(43,5 \pm 7,3)$ foi estatisticamente similar a de $1,5 \mathrm{~mm}(42,1 \pm 6,7)$ e maior que a espessura de $2,0 \mathrm{~mm}(38,0 \pm 7,2)(p=0,001)$. Os menores valores de $R U \mu C$ foram observados para a translucidez LT $(39,0 \pm 7,5)$ e os maiores para a MT $(42,6 \pm 6,9)$. HT $(41,9 \pm 7,4)$ não diferiu estatisticamente de $L T$ e $M T(p=0,028)$. Os valores de $R U \mu C$ e irradiância foram significativamente influenciados pelo tipo de fonte de luz, espessura e translucidez. O Bluephase G2 permitiu maior de passagem de luz em relação ao Radii-cal.

Palavras-chave: Cerâmica, Cimento resinoso, Microcisalhamento, Irradiância.

\section{Introdução}

Os sistemas cerâmicos têm sido amplamente utilizados na Odontologia devido, principalmente, às suas características estéticas e mecânicas. O sucesso clínico das restaurações cerâmicas está diretamente ligado ao procedimento de cimentação. Neste contexto, cada sistema cerâmico tem um protocolo de cimentação específico, o qual está intrinsicamente relacionado com (1) características como espessura, cor e translucidez; (2) mecanismo de polimerização do cimento resinoso; (3) características do aparelho de fotoativação, tais como intensidade de luz e comprimento de onda.

\section{Resultados e Discussão}

Noventa discos de cerâmica (12 mm diâmetro) foram separados em 18 grupos $(n=5)$ de acordo com a espessura $(0,5 ; 1,5$ e 2,0 mm), translucidez (HT, MT e LT) e fonte de luz (Radii-Cal e Bluephase G2) Um lado do disco recebeu acabamento, polimento e glaze. A irradiância $\left(\mathrm{mW} / \mathrm{cm}^{2}\right)$ foi avaliada com potenciômetro Ophir 10 $0^{\mathrm{a}}-\mathrm{V} 2-\mathrm{SH}$ imediatamente após a interposição cerâmica. A RU $\mu \mathrm{C}$ foi realizada à velocidade de 1,0 $\mathrm{mm} / \mathrm{minuto}$ até ocorrer falha. Os dados foram submetidos à Análise de Variância 3 fatores e ao teste de Tukey $(\alpha=0,05)$.

Os valores de RU $\mathrm{RC}$ (MPa) dos grupos RD $(37,1 \pm 6,4)$ apresentaram valores estatisticamente menores que os grupos $B L(45,3 \pm 5,8)(p=0,000)$. A espessura de $0,5 \mathrm{~mm}$ $(43,5 \pm 7,3)$ foi estatisticamente similar a de $1,5 \mathrm{~mm}$ $(42,1 \pm 6,7)$ e maior que a espessura de $2,0 \mathrm{~mm}(38,0 \pm 7,2)$ $(p=0,001)$. Os valores de irradiância estão descritos na tabela 1.
Tabela 1 - Média e desvio padrão da irradiância $\left(\mathrm{mW} / \mathrm{cm}^{2}\right)$ em função da fonte de luz, translucidez e espessura da cerâmica IPS e.max Press.

\begin{tabular}{ccccc}
\hline $\begin{array}{c}\text { Fonte de } \\
\text { luz }\end{array}$ & Espessura & HT & MT & LT \\
\hline Radii Cal & $0,5 \mathrm{~mm}$ & $\begin{array}{c}600,9 \pm 23,4 \\
\mathrm{aA}^{*}\end{array}$ & $\begin{array}{c}496,3 \pm 37,1 \\
\mathrm{aB}^{*}\end{array}$ & $\begin{array}{c}517,2 \pm 20,4 \\
\mathrm{aB}^{*}\end{array}$ \\
& $1,5 \mathrm{~mm}$ & $\begin{array}{c}517,5 \pm 38,9 \\
\mathrm{bA}^{*}\end{array}$ & $\begin{array}{c}456,6 \pm 24,7 \\
\mathrm{abB}\end{array}$ & $\begin{array}{c}458,4 \pm 15,3 \\
\mathrm{bB}^{*}\end{array}$ \\
& $2,0 \mathrm{~mm}$ & $\begin{array}{c}487,5 \pm 32,3 \\
\mathrm{bA}^{*}\end{array}$ & $\begin{array}{c}421,2 \pm 20,4 \\
\mathrm{bB}^{*}\end{array}$ & $\begin{array}{c}386,8 \pm 45,8 \\
\mathrm{cB}\end{array}$ \\
\hline \multirow{2}{*}{$\begin{array}{c}\text { Bluephase } \\
\text { G2 }\end{array}$} & $0,5 \mathrm{~mm}$ & $\begin{array}{c}815,7 \pm 14,9 \\
\mathrm{aA}\end{array}$ & $\begin{array}{c}723,4 \pm 43,8 \\
\mathrm{aB}\end{array}$ & $\begin{array}{c}813,7 \pm 7,0 \\
\mathrm{aA}\end{array}$ \\
& $1,5 \mathrm{~mm}$ & $\begin{array}{c}655,5 \pm 53,5 \\
\mathrm{bA}\end{array}$ & $\begin{array}{c}615,6 \pm 13,3 \\
\mathrm{bA}\end{array}$ & $\begin{array}{c}631,8 \pm 54,6 \\
\mathrm{bA}\end{array}$ \\
& $2,0 \mathrm{~mm}$ & $\begin{array}{c}621,4 \pm 53,5 \\
\mathrm{bA}\end{array}$ & $\begin{array}{c}532,4 \pm 40,1 \\
\mathrm{cB}\end{array}$ & $\begin{array}{c}427,6 \pm 33,8 \\
\mathrm{cC}\end{array}$ \\
\hline Medias seguidas de letras distintas diferem entre si $(\mathrm{p}<0,05)$. & Letras minúsculas
\end{tabular}

Medias seguidas de letras distintas diferem entre si $(p<0,05)$. Letras minúsculas comparam espessura dentro de cada translucidez e fonte de luz. Letras maiúsculas comparam translucidez dentro de cada espessura e fonte de luz. Asteriscos (*) representam diferença estatisticamente significativa entre as fontes de luz para cada espessura e translucidez. Houve diferença tripla significativa (fonte de luz $x$ espessura $x$ translucide $z)(p=0,002)$.

\section{Conclusões}

Os valores de $\mathrm{RU \mu \textrm {C }} \mathrm{e}$ irradiância foram significativamente influenciados pelo tipo de fonte de luz, espessura e translucidez. O Bluephase G2 permitiu maior de passagem de luz em relação ao Radii-cal.

\section{Agradecimentos}

Ao Programa Institucional de Bolsas de Iniciação Científica da Ao Programa Institucional de Bolsas de Iniciação Científica da UNICAMP (PIBIC-CNPq).

${ }^{1}$ Castro HL, Passos SP, Zogheib LV, Bona AD. Effect of cement shade and lightcuring unit on bond strength of a ceramic cemented to dentin. J Adhes Dent. 2012; 14: 155-60 